\title{
Linear Preservers of Perimeters of Nonnegative Real Matrices
}

\author{
SEOK-Zun SONG \\ Department of Mathematics, Cheju National University, Jeju 690-756, Korea \\ e-mail : szsong@cheju.ac.kr \\ KYUNG-TAE KANG \\ Department of Mathematics, Cheju National University, Jeju 690-756, Korea \\ e-mail : kangkt@cheju.ac.kr
}

\begin{abstract}
For a nonnegative real matrix $A$ of $\operatorname{rank} 1, A$ can be factored as $\mathbf{a b}^{t}$ for some vectors $\mathbf{a}$ and $\mathbf{b}$. The perimeter of $A$ is the number of nonzero entries in both $\mathbf{a}$ and $\mathbf{b}$. If $B$ is a matrix of rank $k$, then $B$ is the sum of $k$ matrices of rank 1 . The perimeter of $B$ is the minimum of the sums of perimeters of $k$ matrices of rank 1 , where the minimum is taken over all possible rank-1 decompositions of $B$. In this paper, we obtain characterizations of the linear operators which preserve perimeters 2 and $k$ for some $k \geq 4$. That is, a linear operator $T$ preserves perimeters 2 and $k(\geq 4)$ if and only if it has the form $T(A)=U A V$, or $T(A)=U A^{t} V$ with some invertible matrices $U$ and $V$.
\end{abstract}

\section{Introduction}

There is much literature on the study of linear operators that preserve the ranks of matrices over several semirings([1]-[8]). Nonnegative matrices also have been the subject of research by many authors([2], [5], [6], [8]). Beasley, Gregory and Pullman [2] obtained characterizations of linear operators which preserve the rank of nonnegative real matrices. In [8], Song and Hwang characterized spanning column ranks and their preservers of nonnegative matrices. Beasley, Song, Kang and Sarma [5] treated column ranks of nonnegative real matrices and characterized their preservers.

But there are few papers on the characterizations of linear operators preserving the perimeter of matrices. Beasley et al. characterized those linear operators preserving the rank and perimeter of Boolean rank-1 matrices([1]).

In this paper, we consider the set of linear operators that preserve the perimeter of matrices of rank $k(\geq 2)$ over the nonnegative reals.

Received June 9, 2007.

2000 Mathematics Subject Classification: 15A48, 15A04, 15A23.

Key words and phrases: rank, perimeter, linear operator, $(U, V)$-operator. 


\section{Preliminaries and some results}

Let $M_{m, n}\left(R_{+}\right)$denote the set of all $m \times n$ matrices with entries in $R_{+}$, the set of nonnegative reals. Addition, multiplication by scalars, and the product of matrices are also defined as if $R_{+}$were a field. Throughout this paper, we shall adopt the convention that $1 \leq m \leq n$ unless otherwise specified.

The rank or factor rank, $r(A)$, of a nonzero matrix $A \in M_{m, n}\left(R_{+}\right)$is defined as the least integer $k$ for which there exist $m \times k$ and $k \times n$ matrices $B$ and $C$ with $A=B C$. The rank of a zero matrix is zero.

The Boolean algebra consists of the set $B=\{0,1\}$ equipped with two binary operations, addition and multiplication. The operations are defined as usual except that $1+_{B} 1=1$. If $A=\left[a_{i j}\right]$ is any matrix in $M_{m, n}\left(R_{+}\right)$, we define $A^{*}=\left[a_{i j}^{*}\right]$ to be the $m \times n$ Boolean matrix whose $(i, j)$-th entry is 1 if and only if $a_{i j} \neq 0$. Then ${ }^{*}$ maps $M_{m, n}\left(R_{+}\right)$onto $M_{m, n}(B)$, and preserves matrix addition, product, and multiplication by scalars. That is, ${ }^{*}$ is a homomorphism. It follows that

$$
(A+B)^{*}=A^{*}+B^{*} \text { and }(B C)^{*}=B^{*} C^{*},
$$

for all $A, B \in M_{m, n}\left(R_{+}\right)$and all $C \in M_{n, r}\left(R_{+}\right)$.

If $A \in M_{m, n}\left(R_{+}\right)$with $r(A)=1$, there exist nonzero vectors a $\in M_{m, 1}\left(R_{+}\right)$ and $\mathbf{b} \in M_{n, 1}\left(R_{+}\right)$such that $A=\mathbf{a b}^{t}$. But these vectors $\mathbf{a}$ and $\mathbf{b}$ are not uniquely determined by $A$. For any vector $\mathbf{u} \in M_{m, 1}\left(R_{+}\right),|\mathbf{u}|$ denote the number of nonzero entries in $\mathbf{u}$.

Lemma 2.1. For any factorization $\mathbf{a b}^{t}$ of $A \in M_{m, n}\left(R_{+}\right)$with $r(A)=1,|\mathbf{a}|$ and | $\mathbf{b} \mid$ are uniquely determined by $A$.

Proof. It follows from (2.1) and the fact that $\left|\mathbf{a}^{*}\right|$ and $\left|\mathbf{b}^{*}\right|$ are uniquely determined by $A^{*}$.

Let $A$ be a rank-1 matrix in $M_{m, n}\left(R_{+}\right)$. We define the perimeter of a rank-1 matrix $A, p(A)$, as $|\mathbf{a}|+|\mathbf{b}|$ for arbitrary factorization $A=\mathbf{a b}^{t}$. By Lemma 2.1, $p(A)$ is well-defined. For any matrix $A$ in $M_{m, n}\left(R_{+}\right)$, a rank-1 decomposition of $A$ is a sum of matrices of rank 1 which equals $A$. We say that $A$ has 1 -rank $k$ if $k$ is the minimal number of terms in any rank-1 decomposition of $A$. This shows that $A$ has 1-rank $k$ if and only if $r(A)=k$. If $A \in M_{m, n}\left(R_{+}\right)$, the perimeter of $A, p(A)$, is defined as

$$
\min \left\{\sum_{i=1}^{t} p\left(A_{i}\right) \mid \sum_{i=1}^{t} A_{i} \text { is a rank-1 decomposition of } A, \quad 1 \leq t \leq m\right\} .
$$

Let $P_{k}$ denote the set of matrices in $M_{m, n}\left(R_{+}\right)$whose perimeter is $k$ for $k=$ $2,3, \cdots, m(n+1)$. Let $E_{i j}$ denote the $m \times n$ matrix whose $(i, j)$ th entry is 1 and whose other entries are all 0 , and $E_{m, n}=\left\{E_{i j} \mid 1 \leq i \leq m, 1 \leq j \leq n\right\}$. We call $E_{i j}$ a cell and $\alpha E_{i j}$ a weighted cell for any nonzero scalar $\alpha \in \bar{R}_{+}$. Thus, we have $A \in P_{2}$ if and only if $A$ is a weighted cell. 
For any matrix $A \in M_{m, n}\left(R_{+}\right)$, a line is a row or a column of $A$. The set of cells is collinear if they are located in a same line. We denote the number of all nonzero entries in a matrix $A \in M_{m, n}\left(R_{+}\right)$as $|A|$.

Let $A$ be a matrix in $P_{2 h}(1 \leq h \leq m)$ which has minimum nonzero entries. What is the value of $|A|$ ? The answer is $|A|=h$, and any two nonzero entries are not located in a same line. Let $B$ be a matrix in $P_{2 h+1}(1 \leq h \leq m-1)$ which has minimum nonzero entries. Then we have $|B|=h+1$, and $h$ lines contain all $h+1$ nonzero entries. Furthermore, at least one line contains two nonzero entries. This proves the following Lemma:

Lemma 2.2. The following statements hold:

(1) Let $k=2 h$ with $1 \leq h \leq m$. Then the elements of $P_{k}$ with minimum weighted cells have exactly $h$ nonzero entries, no two of them are in one line.

(2) Let $k=2 h+1$ with $1 \leq h \leq m-1$. Then the elements of $P_{k}$ with minimum weighted cells have exactly $h+1$ nonzero entries which are located in $h$ lines, and at least one line has two nonzero entries.

Consider a matrix

$$
A=\left[\begin{array}{ccc}
a & 0 & b \\
0 & 0 & 0 \\
c & 0 & d
\end{array}\right] \in M_{3,3}\left(R_{+}\right),
$$

where $a b c d \neq 0$. Then the minimum number of lines that contain all the nonzero entries of $A$ is 2. The Lemma 2.3(below) shows that the value of $p(A)$ is either 4 or 6 . In fact, if $a d=b c$, then $p(A)=4$, while $a d \neq b c$ implies $p(A)=6$ from the following factorizations:

$$
A=\left[\begin{array}{l}
a \\
0 \\
c
\end{array}\right]\left[\begin{array}{lll}
1 & 0 & \frac{b}{a}
\end{array}\right] \text { and } A=\left[\begin{array}{l}
1 \\
0 \\
0
\end{array}\right]\left[\begin{array}{lll}
a & 0 & b
\end{array}\right]+\left[\begin{array}{l}
0 \\
0 \\
1
\end{array}\right]\left[\begin{array}{lll}
c & 0 & d
\end{array}\right],
$$

respectively.

Lemma 2.3. Let $A$ be a matrix in $M_{m, n}\left(R_{+}\right)$with $|A|=t+2$. If the minimum number of lines that contain all the nonzero entries of $A$ is $t$, we have either $p(A)=2 t$ or $p(A)=2 t+2$.

Proof. Since $t$ is the minimum number of lines that contain all nonzero entries of $A=\left[a_{i j}\right]$, without loss of generality we may assume $a_{i i} \neq 0$ for $i=1, \cdots, t$. It follows that the remaining two nonzero entries, $x$ and $y$, lie on the $i$ th row or the $j$ th column for some $i, j=1, \cdots, t$. Then two possibilities exist: (a) $x$ and $y$ lie on the same line; (b) $x$ and $y$ lie on two different lines. For the case (a), we have $p(A)=2 t+2$. For the case (b), if there exist distinct indices $i, j$ in $\{1, \cdots, t\}$ with $x=a_{i j}$ and $y=a_{j i}$ such that $\frac{a_{i i}}{a_{j i}}=\frac{a_{i j}}{a_{j j}}$, then we have $p(A)=2 t$. For the other cases, the value of $p(A)$ is $2 t+2$. 


\section{Linear operators preserving $P_{2}$ and $P_{k}$}

In this section, we will characterize those linear operators that preserve perimeters 2 and $k \geq 3$. An $n \times n$ nonnegative real matrix $A$ is said to be invertible if there exists a matrix $B \in M_{n, n}\left(R_{+}\right)$such that $A B=B A=I_{n}$, where $I_{n}$ is the $n \times n$ identity matrix. It is well known [2] that a square matrix $A$ over $R_{+}$is invertible if and only if some permutation of its rows is a diagonal matrix all of whose diagonal entries are nonzero in $R_{+}$.

Lemma 3.1. The perimeter of a matrix of rank 1 is preserved under pre or postmultiplication by an invertible matrix.

Proof. If $A$ is a matrix in $M_{m, n}\left(R_{+}\right)$with $r(A)=1$, there exist nonzero vectors $\mathbf{a} \in M_{m, 1}\left(R_{+}\right)$and $\mathbf{b} \in M_{n, 1}\left(R_{+}\right)$such that $A=\mathbf{a b}^{t}$. Let $U$ and $V$ be invertible matrices in $M_{m, m}\left(R_{+}\right)$and $M_{n, n}\left(R_{+}\right)$, respectively. Then we have

$$
\begin{aligned}
p(U A V) & =p\left(U \mathbf{a b}^{t} V\right)=p\left((U \mathbf{a})\left(V^{t} \mathbf{b}\right)^{t}\right) \\
& =|U \mathbf{a}|+\left|V^{t} \mathbf{b}\right|=|\mathbf{a}|+|\mathbf{b}|=p(A) .
\end{aligned}
$$

The Lemma now follows.

A mapping $T: M_{m, n}\left(R_{+}\right) \rightarrow M_{m, n}\left(R_{+}\right)$is called a linear operator if $T(\alpha A+\beta B)=\alpha T(A)+\beta T(B)$ for all $A, B \in M_{m, n}\left(R_{+}\right)$and for all $\alpha, \beta \in R_{+}$. For a linear operator $T$ on $M_{m, n}\left(R_{+}\right)$, we say that $T$ is a $(U, V)$-operator if there exist invertible matrices $U \in M_{m, m}\left(R_{+}\right)$and $V \in M_{n, n}\left(R_{+}\right)$such that $T(A)=U A V$ for all $A$ in $M_{m, n}\left(R_{+}\right)$, or $m=n$ and $T(A)=U A^{t} V$ for all $A$ in $M_{m, n}\left(R_{+}\right)$. A linear operator $T$ on $M_{m, n}\left(R_{+}\right)$is said to preserve perimeter if $p(T(A))=p(A)$ for all $A \in M_{m, n}\left(R_{+}\right)$. A linear operator $T$ on $M_{m, n}\left(R_{+}\right)$preserves $P_{k}$ if $T(A) \in P_{k}$ whenever $A \in P_{k}$.

Proposition 3.2. If $T$ is a $(U, V)$-operator on $M_{m, n}\left(R_{+}\right)$, then $T$ preserves perimeter.

Proof. Since $T$ is a $(U, V)$-operator, there exist invertible matrices $U \in M_{m, m}\left(R_{+}\right)$ and $V \in M_{n, n}\left(R_{+}\right)$such that either $T(A)=U A V$, or $m=n$ and $T(A)=U A^{t} V$ for all $A$ in $M_{m, n}\left(R_{+}\right)$. Let $A=\sum_{i=1}^{t} A_{i}$ be a rank-1 decomposition of $A$.

For the case $T(A)=U A V$, we have

$$
\sum_{i=1}^{t} p\left(T\left(A_{i}\right)\right)=\sum_{i=1}^{t} p\left(U A_{i} V\right)=\sum_{i=1}^{t} p\left(A_{i}\right)
$$

by Lemma 3.1. This implies that $p(T(A))=p(A)$. For the case $T(A)=U A^{t} V$, we can show that $p(T(A))=p(A)$ by the similar method as above. Thus the proof is complete.

Let $R_{i}=\left\{E_{i j} \mid 1 \leq j \leq n\right\}, C_{j}=\left\{E_{i j} \mid 1 \leq i \leq m\right\}, \mathbf{R}=\left\{R_{i} \mid 1 \leq i \leq m\right\}$ and $\mathbf{C}=\left\{C_{j} \mid 1 \leq j \leq n\right\}$. For a linear operator $T$ on $M_{m, n}\left(R_{+}\right)$, define $T^{*}(X)=$ 
$[T(X)]^{*}$ for all $X$ in $M_{m, n}\left(R_{+}\right)$. Let $T^{*}\left(R_{i}\right)=\left\{T^{*}\left(E_{i j}\right) \mid 1 \leq j \leq n\right\}$ for each $i=1, \cdots, m$ and $T^{*}\left(C_{j}\right)=\left\{T^{*}\left(E_{i j}\right) \mid 1 \leq i \leq m\right\}$ for each $j=1, \cdots, n$. Let $T$ be a linear operator on $M_{m, n}\left(R_{+}\right)$which preserves $P_{2}$. Then $T^{*}$ maps $E_{m, n}$ into $E_{m, n}$ because $P_{2}$ is the set of all weighted cells.

Lemma 3.3. Let $4 \leq k \leq 2 m$. If $T$ is a linear operator on $M_{m, n}\left(R_{+}\right)$which preserves $P_{2}$ and $P_{k}$, then $T^{*}$ maps $E_{m, n}$ onto $E_{m, n}$.

Proof. We consider two cases: (a) $k=2 h+1$ with $2 \leq h \leq m-1$ and (b) $k=2 h$ with $2 \leq h \leq m$.

Case a. $k=2 h+1$ with $2 \leq h \leq m-1$. Suppose $T^{*}$ does not map $E_{m, n}$ onto $E_{m, n}$. Then there exist two distinct cells $E_{i j}$ and $E_{p q}$ in $E_{m, n}$ such that $T^{*}\left(E_{i j}\right)=T^{*}\left(E_{p q}\right)$. By Lemma 2.2-(2), there is a matrix $X=\left[x_{i j}\right] \in P_{k}$ which has minimum nonzero entries such that $x_{i j} \neq 0$ and $x_{p q} \neq 0$. But then we have $T(X) \notin P_{k}$ because $|T(X)|<|X|$. This contradiction shows that $T^{*}$ maps $E_{m, n}$ onto $E_{m, n}$.

Case b. $k=2 h$ with $2 \leq h \leq m$. Assume that $T^{*}\left(E_{i j}\right)=T^{*}\left(E_{p q}\right)$ for two distinct cells $E_{i j}$ and $E_{p q}$ in $E_{m, n}$. If $E_{i j}$ and $E_{p q}$ are not collinear, by the similar argument of case a) with Lemma 2.2-(1), there exists a matrix $Y=\left[y_{i j}\right]$ in $P_{k}$ which has minimum nonzero entries such that $y_{i j} \neq 0$ and $y_{p q} \neq 0$. But then we have $T(Y) \notin P_{k}$ because $|T(Y)|<|Y|$. This is a contradiction. Hence $E_{i j}$ and $E_{p q}$ are collinear. Without loss of generality we may assume $(i, j)=(1,1)$ and $(p, q)=(1,2)$.

Consider a matrix $Z=E_{1,1}+E_{1,2}+E_{1,3}+\sum_{i=2}^{h-1} E_{i, i} \in M_{m, n}\left(R_{+}\right)$. Then we have $Z \in P_{k}$ with $|Z|=h+1$. But then $|T(Z)| \leq h$ follows from $T^{*}\left(E_{i j}\right)=T^{*}\left(E_{p q}\right)$. Since a matrix $A \in P_{k}$ with minimum nonzero entries contains $h$ nonzero entries, and $T$ preserves $P_{k}$, we have $|T(Z)|=h$. By Lemma 2.2-(1), any two nonzero entries in $T(Z)$ are not collinear. Without loss of generality we may assume that $T^{*}\left(E_{1,1}\right)=T^{*}\left(E_{1,2}\right)=E_{1,1}, T^{*}\left(E_{i, i}\right)=E_{i, i}$ for $2 \leq i \leq h-1$ and $T^{*}\left(E_{1,3}\right)=E_{r, s}$, where $r, s \geq h$.

Let $W=E_{1,1}+E_{1,3}+E_{1, t}+\sum_{i=2}^{h-1} E_{i, i}$ be a matrix in $M_{m, n}\left(R_{+}\right)$with $4 \leq t \leq n$ so that $W \in P_{k}$. Suppose that $T^{*}\left(E_{1, t}\right)$ is neither $E_{i, i}$ for $1 \leq i \leq h-1$ nor $E_{r, s}$. Then we have $p(T(W)) \geq k+1$, a contradiction because $T$ preserves $P_{k}$. Hence for all $4 \leq t \leq n, T^{*}\left(E_{1, t}\right)$ is one of $E_{i, i}$ for $1 \leq i \leq h-1$ or $E_{r, s}$. Let $S=E_{1,3}+E_{1,4}+E_{1, h}+\sum_{i=2}^{h-1} E_{i, i}$ be a matrix in $M_{m, n}\left(R_{+}\right)$so that $S \in P_{k}$. But then we have $|T(S)| \leq h-1$, and hence $T(S) \notin P_{k}$, a contradiction. This completes the proof.

Let $T: M_{m, n}\left(R_{+}\right) \rightarrow M_{m, n}\left(R_{+}\right)$be a linear operator which preserves $P_{2}$. 
Because $T^{*}$ maps $E_{m, n}$ into $E_{m, n}$, we can write for all $X=\left[x_{i j}\right] \in M_{m, n}\left(R_{+}\right)$,

$$
T(X)=\sum_{i=1}^{m} \sum_{j=1}^{n} x_{i j} b_{i j} T^{*}\left(E_{i j}\right)
$$

where each $b_{i j}$ is a nonzero scalar in $R_{+}$.

Lemma 3.4. Let $k=2 h$ with $2 \leq h \leq m$. If $T$ is a linear operator on $M_{m, n}\left(R_{+}\right)$ which preserves $P_{2}$ and $P_{k}$, then $T$ maps lines onto lines.

Proof. It follows from Lemma 3.3 that $T^{*}$ maps $E_{m, n}$ onto $E_{m, n}$. Suppose $T$ does not preserve lines to lines. Then there exist two distinct cells $E_{i j}$ and $E_{p q}$ which are not collinear such that $T\left(E_{i j}\right)$ and $T\left(E_{p q}\right)$ are collinear. Let

$$
X=E_{i j}+E_{p q}+\sum_{t=1}^{h-2} E_{i_{t} j_{t}}
$$

be a matrix in $M_{m, n}\left(R_{+}\right)$, where all $i_{t}, i, p$ are distinct and all $j_{t}, j, q$ are distinct. Then we have $p(X)=k$, while $p(T(X)) \leq 3+2(h-2)=2 h-1<k$, a contradiction. Therefore $T$ maps lines onto lines.

Lemma 3.5. Let $4 \leq k \leq 2 m$. If $T$ is a linear operator on $M_{m, n}\left(R_{+}\right)$which preserves $P_{2}$ and $P_{k}$, then $T$ maps lines onto lines.

Proof. By Lemma 3.4, we can assume that $k=2 h+1$ with $2 \leq h \leq m-1$. Lemma 3.3 implies that $T^{*}$ maps $E_{m, n}$ onto $E_{m, n}$. If $T$ does not map lines onto lines, without loss of generality we may assume that $T\left(E_{1,1}\right)$ and $T\left(E_{1,2}\right)$ are not collinear. Let $X=E_{1,1}+E_{1,2}+\sum_{i=2}^{h} E_{i, i}$ be a matrix in $M_{m, n}\left(R_{+}\right)$so that $X \in P_{k}$. But then $T(X)$ is a sum of $h+1$ weighted cells with $p(T(X))=k$ because $T$ preserves $P_{k}$. By Lemma 2.2-(2), all nonzero entries in $T(X)$ must be located in $h$ line, and at least one line has two nonzero entries.

Let $Y$ be a matrix which is the sum of $h$ cells including $E_{1,1}$ and $E_{1,2}$ such that $h$ is the minimum number of lines that contain all the nonzero entries of $T(Y)$. Let $Z=Y+E_{1,3}+E_{1, h}$ be a matrix in $M_{m, n}\left(R_{+}\right)$so that $p(Z)=5+2(h-2)=$ $2 h+1=k$. But then $T(Z)$ is the sum of $h+2$ weighted cells, and the minimum number of lines that contain all the nonzero entries of $T(Z)$ is greater than or equal to $h$. By Lemma 2.3, the perimeter of $T(Z)$ is either $2 h, 2 h+2,2 h+3$ or $2 h+4$, a contradiction. Therefore $T$ maps lines onto lines.

Theorem 3.6. If $T$ is a linear operator on $M_{m, n}\left(R_{+}\right)$which preserves $P_{2}$ and $P_{k}$ with $4 \leq k \leq \min (2 m, n+2)$, we have $T$ is a $(U, V)$-operator.

Proof. It follows from Lemma 3.3 that $T^{*}$ maps $E_{m, n}$ onto $E_{m, n}$. By Lemma 3.5, there are two cases; (a) $T^{*}$ maps $\mathbf{R}$ onto $\mathbf{R}$ and maps $\mathbf{C}$ onto $\mathbf{C}$ or (b) $T^{*}$ maps $\mathbf{R}$ onto $\mathbf{C}$ and $\mathbf{C}$ onto $\mathbf{R}$.

Case a. We note that $T^{*}\left(R_{i}\right)=R_{\sigma(i)}$ and $T^{*}\left(C_{j}\right)=C_{\tau(j)}$ for all $i, j$, where $\sigma$ 
and $\tau$ are permutations of $\{1, \cdots, m\}$ and $\{1, \cdots, n\}$, respectively. Let $P$ and $Q$ be the permutation matrices corresponding to $\sigma$ and $\tau$, respectively. Then for any $E_{i j} \in E_{m, n}$, we can write $T\left(E_{i j}\right)=b_{i j} E_{\sigma(i) \tau(j)}$ for some nonzero scalar $b_{i j} \in R_{+}$. Now we claim that for all $i, l \in\{1, \cdots, m\}$ and all $j, r \in\{1, \cdots, n\}$,

$$
\frac{b_{i j}}{b_{i r}}=\frac{b_{l j}}{b_{l r}}
$$

Consider a matrix

$$
A=E_{i j}+E_{i r}+E_{l j}+E_{l r}+\sum_{t=1}^{k-4}\left(E_{i j_{t}}+E_{l j_{t}}\right),
$$

where $j_{t} \neq j, r$ for all $t=1, \cdots, k-4$. Then we have $p(A)=k$ and the image of $A$ becomes

$$
\begin{aligned}
T(A) & =b_{i j} E_{\sigma(i) \tau(j)}+b_{i r} E_{\sigma(i) \tau(r)}+b_{i j} E_{\sigma(i) \tau(j)}+b_{i r} E_{\sigma(i) \tau(r)} \\
& +\sum_{t=1}^{k-4}\left(b_{i j_{t}} E_{\sigma(i) \tau\left(j_{t}\right)}+b_{l j_{t}} E_{\sigma(l) \tau\left(j_{t}\right)}\right) .
\end{aligned}
$$

Since $T(A)$ has perimeter $k$, it follows that $\frac{b_{i j}}{b_{i r}}=\frac{b_{l j}}{b_{l r}}=\frac{b_{i j_{t}}}{b_{l j_{t}}}$ for all $t=1, \cdots, k-4$. Let $C \in M_{m, m}\left(R_{+}\right)$and $D \in M_{n, n}\left(R_{+}\right)$be diagonal matrices such that

$$
c_{11}=1, \quad d_{11}=b_{11}, \quad c_{i i}=\frac{b_{i 1}}{b_{11}}, \quad \text { and } \quad d_{j j}=b_{1 j}
$$

for all $i=2, \cdots, m$ and $j=2, \cdots, n$. Then we have $b_{i j}=c_{i i} d_{j j}$ for all $i=1, \cdots, m$ and $j=1, \cdots, n$. Let $X=\left[x_{i j}\right]$ be any $m \times n$ matrix in $M_{m, n}\left(R_{+}\right)$. Then we have

$$
\begin{aligned}
T(X) & =T\left(\sum_{i=1}^{m} \sum_{j=1}^{n} x_{i j} E_{i j}\right)=\sum_{i=1}^{m} \sum_{j=1}^{n} x_{i j} T\left(E_{i j}\right) \\
& =\sum_{i=1}^{m} \sum_{j=1}^{n} x_{i j} b_{i j} E_{\sigma(i) \tau(j)}=\sum_{i=1}^{m} \sum_{j=1}^{n} c_{i i} x_{i j} E_{\sigma(i) \tau(j)} d_{j j} \\
& =C P X Q D .
\end{aligned}
$$

Since $C P=U$ is an $m \times m$ invertible matrix and $Q D=V$ is an $n \times n$ invertible matrix, it follows that $T$ is a $(U, V)$-operator.

Case b. This is the case of $m=n$ and $T^{*}\left(R_{i}\right)=C_{\sigma(i)}$ and $T^{*}\left(C_{j}\right)=R_{\tau(j)}$ for all $i$ and $j$, where $\sigma$ and $\tau$ are permutations of $\{1, \cdots, m\}$. By similar argument to case a), we obtain that $T(X)$ is of the form $T(X)=C P X^{t} Q D$. Thus $T$ is a $(U, V)$-operator.

We say that a linear operator $T$ on $M_{m, n}\left(R_{+}\right)$strongly preserves perimeter $k$ if $T(A) \in P_{k}$ if and only if $A \in P_{k}$. 
Theorem 3.7. If $T$ is a linear operator on $M_{m, n}\left(R_{+}\right)$which strongly preserves $P_{2}$ and $P_{3}, T$ is a $(U, V)$-operator.

Proof. It is obvious that $T^{*}$ maps $E_{m, n}$ onto $E_{m, n}$ because $T$ strongly preserves $P_{2}$. If $T$ does not map lines to lines, there exist two distinct cells $E_{i j}$ and $E_{p q}$ which are collinear such that $T\left(E_{i j}\right)$ and $T\left(E_{p q}\right)$ are not collinear. But then the perimeter of $E_{i j}+E_{p q}$ is 3 , while that of $T\left(E_{i j}+E_{p q}\right)$ is 4 , a contradiction. It follows that $T$ maps lines to lines. By the similar method in the proof of Theorem 3.6, we have $T$ is a $(U, V)$-operator.

Corollary 3.8. Let $T$ be a linear operator on $M_{m, n}\left(R_{+}\right)$. Then the following are equivalent;

(1) $T$ is a $(U, V)$-operator.

(2) $T$ preserves perimeter.

(3) $T$ preserves $P_{2}$ and $P_{k}$ with $4 \leq k \leq \min (2 m, n+2)$.

(4) $T$ strongly preserves $P_{2}$ and $P_{k}$ with $3 \leq k \leq \min (2 m, n+2)$.

Proof. The proof follows from Theorem 3.6 and Theorem 3.7.

Thus we have characterizations of the linear operators which preserve all perimeters over the nonnegative reals.

\section{References}

[1] L. B. Beasley, G. S. Cheon, Y. B. Jun and S. Z. Song, Rank and perimeter preservers of Boolean rank-1 matrices, J. Korean Math. Soc., 41(2004), 397-406.

[2] L. B. Beasley, D. A. Gregory and N. J. Pullman, Nonnegative rank-preserving operators, Linear Algebra Appl., 65(1985), 207-223.

[3] L. B. Beasley and N. J. Pullman, Boolean rank-preserving operators and Boolean rank-1 spaces, Linear Algebra Appl., 59(1984), 55-77.

[4] L. B. Beasley, S. Z. Song and S. G. Lee, Zero term rank preservers, Linear and Multilinear Algebra, 48(2001), 313-318.

[5] L. B. Beasley, S. Z. Song, K. T. Kang and B. K. Sarma, Column ranks and their preservers over nonnegative real matrices, Linear Algebra Appl., to appear.

[6] A. Berman and R. J. Plemmons, Nonegative matrices in the mathematical sciences, Academic, New York (1976).

[7] C. K. Li and S. J. Pierce, Linear preserver problems, Amer. Math. Monthly, 108(2001), 591-605.

[8] S. Z. Song and S. G. Hwang, Spanning column ranks and their preservers of nonnegative matrices, Linear Algebra Appl., 254(1997), 485-495. 